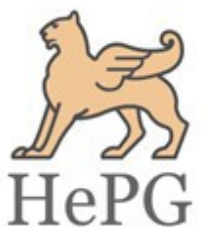

ISSN: 2348-1900

Plant Science Today

http://www.plantsciencetoday.online

\title{
Role of tolerance to resource demand - supply mismatch in a model of annual plants
}

\author{
Michel Droz $^{1^{*}}$ and Andrzej Pękalski ${ }^{2}$ \\ ${ }^{1}$ Department of Theoretical Physics, University of Genèva, 1211 Genève 4, Switzerland \\ ${ }^{2}$ Institute of Theoretical Physics, University of Wrocław, pl. M. Borna 9, 50-203 Wroc ław, Poland
}

Article history

Received: 18 August 2017

Accepted: 31 October 2017

Published: 29 November 2017

(C) Droz and Pękalski (2017)

Editor

K K Sabu

Publisher

Horizon e-Publishing Group

Correspondence

Michel Droz

Мichel.Droz@unige.ch

\begin{abstract}
We propose and discuss a simulation model of annual plants competing for a single resource. Plants are characterised by their tolerance to a surplus of this resource and the maximum number of seeds a plant can produce in a year. Interaction among plants is reduced to blocking a part of the resource by the plant's nearest neighbours. Spatial and temporal conditions are homogeneous. There are no trade-off mechanisms nor immigrants. Plants may suffer from both a lack and too much of the resource. We consider two systems - plants of one type (target plants) and a mixture of two types, where the second type differs from the target ones only by the tolerance to surplus of the resource. We show how the life cycle of a plant depends on its tolerance, on supply of the resource and on how it is affected by the presence of the second type of annuals. We demonstrate that even in such a simple system coexistence of the two species is possible, and we determine the conditions for this. We present also a mean field type approach to the problem, showing that the results from simulations and mean field are quite similar. However the mean field approach cannot answer questions concerning spatial arrangement of plants, like possible formation of niches for different types of plant.
\end{abstract}

Keywords: plant dynamics; Monte Carlo simulations; water supply-demand mismatch; annual plants

Citation: Droz M, Pękalski A. Role of tolerance to resource demand - supply mismatch in a model of annual plants. Plant Science Today 2017;4(4):191-201. doi: 10.14719/pst.2017.4.4.332

\section{Introduction}

The problem of coexistence of species and maintaining biodiversity is one of the central issues of plant ecology (1). As such it has many facets and the number of papers devoted to the subject is enormous. There are many papers discussing the competition - colonisation trade-off (2-6), role of heterogeneity of space $(1,7,8)$, various type of disturbance, such as fire, flooding or grazing (9-11) or tolerance-fecundity trade-off (12). Pairwise (PW) experiments of annual plants have been reported by Goldberg and Fleetwood (13), where the effect of the presence of the nearest neighbouring plants has been studied and the plant types differed in many aspects. The focus was on the role played by the size of the seeds in the competition between plants. The problem of how the presence of one type of plants affects the target plants, has no satisfactory answer (14-17) and therefore further, also theoretical, studies are much needed. We shall consider here mostly the stabilising mechanism (1), i.e. resource partitioning. 
In this paper we present a model of a PW simulation experiment of annual plants. We do not assume, as is quite often done, that competing plants have several features and while one type is superior in one characteristic, it is inferior in another. In short, there is no trade-off mechanism of any kind. The habitat is homogeneous and does not offer by itself a niche more suitable for one type of plant. There is just one resource and there are no immigrants. Finally, while in all papers known to us, only the effect of a shortage of a resource has been studied, we allow for a surplus of the resource, having also a negative effect on the population. To the best of our knowledge such a model has never been studied. The model, described in detail in the next section is of individual based type and its properties are determined by Monte-Carlo type numerical simulations. Next section is devoted to a meanfield approach (MFA) to the same problem. namely:

We revisit some important questions (17),

- In a pairwise evolution which species dominates at a particular time?

- Is coexistence of two species possible, and if so, under what conditions?

- What is the effect of the presence of another species on the performance of the target plants?

- How do the above relations change when the supply of the resource vary - from a shortage, through optimal to a surplus. How the dominance changes with the supply of the resource?

Dominance means here that one species is more abundant than the other (17).

There are many factors characterising a plant community and accounting for even most of them would make the model intractable, banning exploration of the full parameter space. Therefore we have decided to reduce the characteristics of the plants to just their demand for one resource tolerance to a surplus of it and the maximum number of seeds a plants can produce in a year. The term tolerance is used here sensu MullerLandau (12). It means tolerance to stress, here coming from either too much or not enough of the resource.

\section{Model}

We study a homogeneous habitat composed of a population of annual plants living on plaquettes of a square lattice of size $L \times L$ with hard boundary conditions. In the first part of the paper we shall investigate a system with only one type of annual plant. Here we determine the role of the control parameters and the mechanisms at play. In the second part we study a system with two types of plant and we establish the influence of the presence of the second type of plant on the first. In both cases, a lattice site could be either empty or contain only one plant. Plants are competing for just one resource (water), which comes from outside. Each plant has the same demand for it, which is our yardstick, in the sense that all quantities referring to water, like the rainfall, actual amount of water available to a plant or its tolerance are normalised by this demand, hence they are dimensionless quantities. Tolerance, $\tau$ measures how well a plant copes with surplus of water. Larger value of $\tau$ mean that the plant better supports water supply bigger than the demand, equal 1 by definition, while small $\tau$ characterise plants better supporting shortage of water. The plants of a given type have the same tolerance. The system is homogeneous, i.e. the value of the rainfall is the same on all sites. The structure of our model is, to some extent, based on the papers (18) and (19) where annual plants living in mountain areas have been studied. Authors have shown i.a. that the seeds' success depends strongly on the external conditions and that plants have different tolerances to water. Their coexistence has been observed for a period of several years (18).

In our model initially a given number of plants, 2000 for one type of plants and 1000 of each type when there are two of them, is randomly distributed on the lattice. Increasing the initial number of plants to, say, 5000 is virtually unnoticed after some 10 years. The life cycle of a plant (a year) is composed of the germination and adult phases. In the latter the plant produces seeds, distributes them in a given neighbourhood and then dies. Each year the rainfall, $w$, on each site is the same. Each adult plant interacts with its nearest neighbours (NN) as the roots of the neighbours block a certain amount of water, thus reducing the water supply available to the plant. Root competition and mechanisms inhibiting access of other roots to resources (called sometimes contest competition or allelopathy) is a well known phenomenon (20). Effective amount of water available to a plant $i$ having $n n_{i} \mathrm{NN}$ is

$$
\delta_{i}=w\left(1-\alpha \cdot n n_{i}\right),
$$

where the factor $\alpha$ determines the strength of the interactions among the plants. The value $\alpha=0.1$, which we took for simulations is a compromise between a smaller values when the effect of the interactions is lost and a larger value, when it is overwhelming, reducing the influence of the external conditions (rainfall). In general, reducing the interaction strength leads to an increase of the plants' abundance, sharpening also its peak value and bringing it closer to $\delta=1$, as with weak interactions less water is blocked. Since both types of plant use the same resource, we have the case of $p=1$, i.e. complete niche overlap in the terminology of Chesson (1). Smaller values of $p$ correspond to partial sharing of the resource. 
Our control parameter $\tau$ describes how well a plant copes with too much water. Therefore when the amount of water available to a plant is less than the optimum supply, i.e. when $\delta<1$, large tolerance has a negative effect. When the situation is the opposite - there is too much water, large $\tau$ is beneficial. We may therefore determine the survival chance a plant $i$ of type $m$ has, if $\delta_{i}$ is larger than 1 , as

$$
\pi_{i}(m)=\left\{\begin{array}{l}
\exp \left(-\left(1-\delta_{i}\right)^{2} \cdot \tau(m) / \delta_{i}\right) \text { if } \delta_{i}<1 \\
\exp \left(-\left(1-\delta_{i}\right)^{2} /\left(\tau(m) . \delta_{i}\right)\right) \text { if } \delta_{i}<1
\end{array}\right.
$$

In the biological literature there is no form of the survival chance that could be regarded as a canonical one. We have therefore decided on a simple form which fulfils some natural conditions. It must peak when the supply is equal to to the demand for the resource and should decrease when the supply departs from the optimum, reaching zero if there is no water. The above form agrees with those demands. It should also be mentioned that were we to decide to take another form, as e.g.

$$
\pi_{i} \sim \delta_{i} / \tau(m), \text { for } \pi_{i}<1
$$

and the inverse of it when $\pi_{i}>1$, the results from simulations would be qualitatively quite similar.

A random number $r_{i} \in(0,1)$ is taken from a uniform distribution and if it is smaller than $\pi_{i}(m)$, the plant survived, otherwise it is removed. Once the plant has passed the survival test, it produces seeds, the number of which depends on $\pi_{i}$, and the assumed maximum number of seeds, $\beta$, which a plant can produce in optimal conditions. Using the same function for checking the plant's survival and the number of seeds it produced in a year might be a simplification, however, as shown by (21) the two are closely related and therefore such an assumption is well justified. Therefore the number of seeds produced by the plant $i$ is

$$
s_{i}(m)=E\left[\beta . \pi_{i}(m)\right] .
$$

Where $E[\ldots]$ means the integer part of [...]. The seeds are then dispersed randomly over 13 sites in the neighbourhood of the plant, including the site on which the plant grows. The choice of 13 sites in the nearest neighbourhood minimises the probability that a plant will put most of its seeds on one site, thus reducing greatly their germination success. It is, however, not a crucial parameter and increasing it to, say, 25 sites will not produce visible effects. After dispersing the seeds, plants die and are removed from the system. There is no limitation on the number of seeds which could be on a given site. This terminates the adult phase of the plant's life.
Next comes the germination phase. Each site is visited just once and from it one seed is chosen for germination. It does not therefore really matter whether on a site there are 3 or 15 seeds, since only one is chosen. We realise that this independence of the germination success on the number of seeds in a given site is a simplification. Allowing for the germination success to depend on the number of seeds would introduce additional parameters, which value would be difficult to establish from the field data.

We assume that seedlings have too short roots to block water from the NN, hence there are no interactions among seedlings and their survival chance is calculated from a formula similar to that for adult plants, however with the blocking factor $\alpha$ set to 0

$$
\hat{\pi}(m)=\left\{\begin{array}{l}
\exp \left(-(1-w)^{2} \cdot \tau(m) / w\right) \text { if } w<1 \\
\exp \left(-(1-w)^{2} /(\tau(m) . w)\right) \text { if } w<1
\end{array}\right.
$$

Once all sites with seeds have been visited, the seeds are removed from the system - there is no seed bank. Seedlings become adult plants and a new year starts. Plants are randomly chosen, put into the survival test and if they passed it, produce seeds which could germinate etc.

In the following we shall first show how a population of one type of plants with a given tolerance reacts to changes of the external parameter $w$. Afterwards we shall determine the influence the second type of plants has on the target plants.

The two types of plant, called hereafter P1 and P2, could differ in many respects - their size, type and number of seeds, tolerance to surplus of the resource etc. To keep the problem simple we assume that the only difference is the tolerance.

We have the following control parameters reduced resource supply $w$, tolerance of plants $\mathrm{P} 1$ - $\tau_{1}$ and of plants P2 $-\tau_{2}$, maximum number of seeds a plant can produce $\beta$, equal for both types of plant, and the interaction strength $\alpha$. The last two parameters we fix at the following values $\beta=6$ and $\alpha=0.1$. Possible effects of changing theses values are discussed later.

\section{Results}

\subsection{One type of plant}

We consider a square lattice of dimensions $L \times L$ with $L=100$ on which initially 2000 plants of one type are put in random positions. Dynamics has been described in the previous section. We have found that a population of plants reaches a stationary state, where the fluctuations of the number of plants are less than $1 \%$. The number of plants in the stationary state varies very little from one realisation (random initial distribution of the 
same number of plants) to another. We have limited the simulations to 100 years and the data presented are averaged over 50 realisations differing in the initial random distribution of plants. Error bars are smaller than the thickness of the lines.

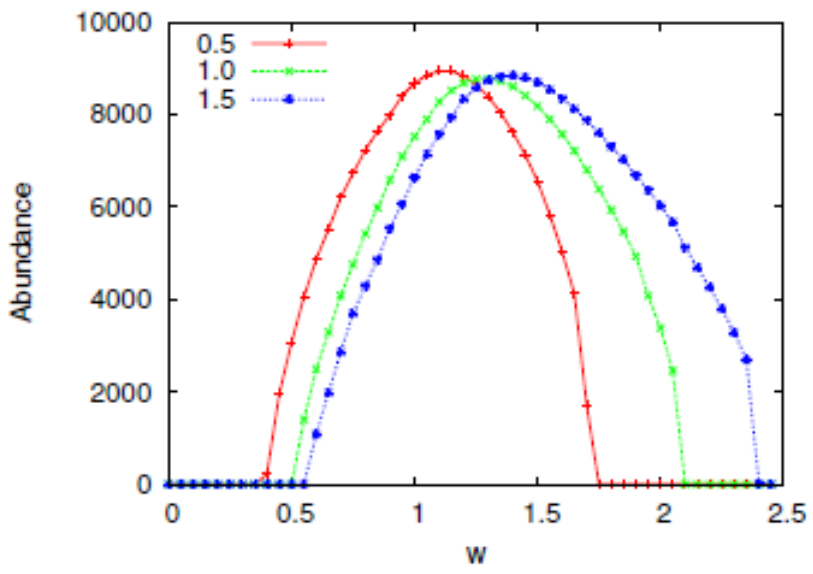

Figure 1: Number of plants P1 for various tolerances as a function of the reduced resource supply $w$. Colour on line.

Figure 1 shows the number of plants as a function of $w$, the resource supply divided by the demand for it, for several values of the tolerance $\tau$. As could be expected, when the tolerance is small $(\tau=0.5)$ plants could live in regions where the water supply is below the optimum. With increasing tolerance, it is more difficult for the plants to live in those "dry regions", but they are now able to grow where the rainfall is larger than the optimum. In some sense we find here the analogues of the $R^{*}$ level introduced by Tilman (25) as the resource level at which the species is just able to persist. In our model we have two such limiting values - the lower level $w_{1}^{*}$ and the upper limit $w_{2}^{*}$. Species could exist within those two borders.

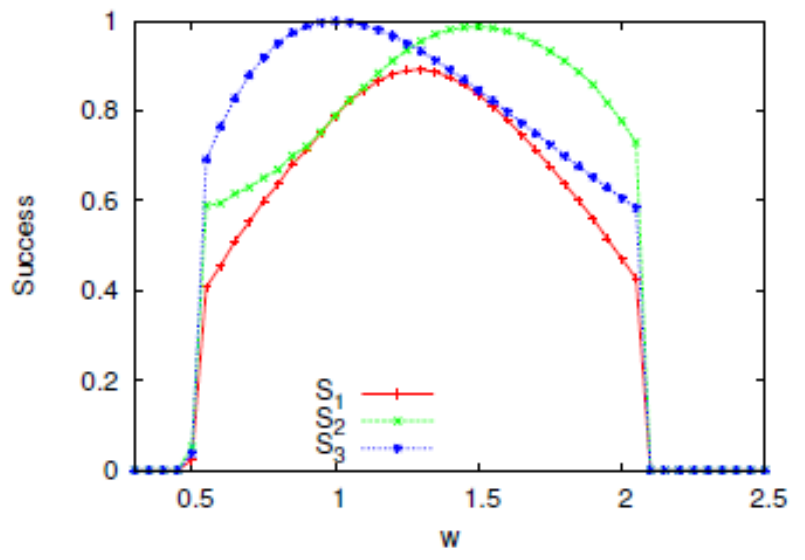

Figure 2: Three successes. S3 - seeds success, S2 - seedlings success, S1 - total success of plants (product of the previous ones) as functions of the resource supply and for $\tau=1.0$. Colour on line.

Figure 2 shows how the three successes that of seeds $\left(S_{3}\right)$, of seedlings $\left(S_{2}\right)$ and the total - of plants $\left(S_{1}\right)$ depend on the rainfall for a tolerance equal 1.0. Other values of tolerance lead to quite similar diagrams. The successes are defined as follows. $S_{3}$ is the ratio of the number of seedlings (i.e. seeds that germinated) in a given year to the number of sites with seeds in the same year. Since from a site only one seed could germinate, it is the number of sites, not the number of seeds, which determines this success. $S_{2}$ is the seedlings success - the ratio of the number of adult plants which were able to produce seeds, to the number of seedlings. Finally, $S_{1}$ is the total success - the ratio of adult plants producing seeds to the number of sites with seeds, or simply $S_{1}=S_{3} \cdot S_{2}$. The presented data come from averaging over the last 20 years before the end of the simulations and over 50 independent realisations.

Since there is no interaction (blocking) among seedlings, the $S_{3}$ curve showing the seeds' success peaks at $\mathrm{w}=\delta=1$, hence at the optimum. Decrease on either side of the maximum is rather fast, since there is no "smoothing" effect of reducing the water available by the NN. For the seedlings the maximum is much wider and less sharp and also shifted to higher than $\mathrm{w}=\tau$ values, since part of the water is now blocked and not all plants can survive till the moment they can produce seeds. Wider maximum comes from the fact that plants have a different number of $\mathrm{NN}$ and therefore the blocking effect for each plant could be different. Finally the total success of plants - i.e. how many seeds were able to germinate and lead to plants which can reproduce, shows a symmetric curve stretching from one maximum (for $S_{3}$ ) to the other one (for $S_{2}$ ).

Spatial arrangement of plants may change in different external conditions, viz. for different $w$. A convenient way to measure it is the densitydensity correlation function $C(r)$, defined as

$$
C(r)=\left\langle\rho\left(r_{0}\right) \rho\left(r_{0}+r\right)\right\rangle,
$$

where $\rho\left(r_{0}\right)$ is the density of plants at site $r_{0}$ and $\rho\left(r_{0}+r\right)$ is the density at a distance $r$ from $r_{0}$. The average is over all plants and different realisations. Assuming that the lattice constant is equal 1, we get the dependence of $\mathrm{C}(r)$ (up to 5-th neighbour) on the distance $r$, as shown in figure 3 for plants with tolerance $\tau=1.0$. For other tolerances the features are similar. The correlation functions $C(r)$ shown in figures 3 have been calculated in three points - (1) when the water is scarce ( $w=0.6),(2)$ when there is as much water as needed $(\mathrm{w}=1.0)$ and (3) when there is too much of water $(\mathrm{w}=1.4)$.

When there is not enough water plants are expected to avoid growing in closely packed clusters, since each NN blocks a certain amount of, already scarce, resource. When the supply of the resource is about what a plant needs, one may 
expect a balance between the average number of nearest and farther neighbours. Finally, when there is too much of the resource, growing in close clusters becomes beneficial, as it allows to reduce the too large supply. This is exactly what is seen in figure 3.

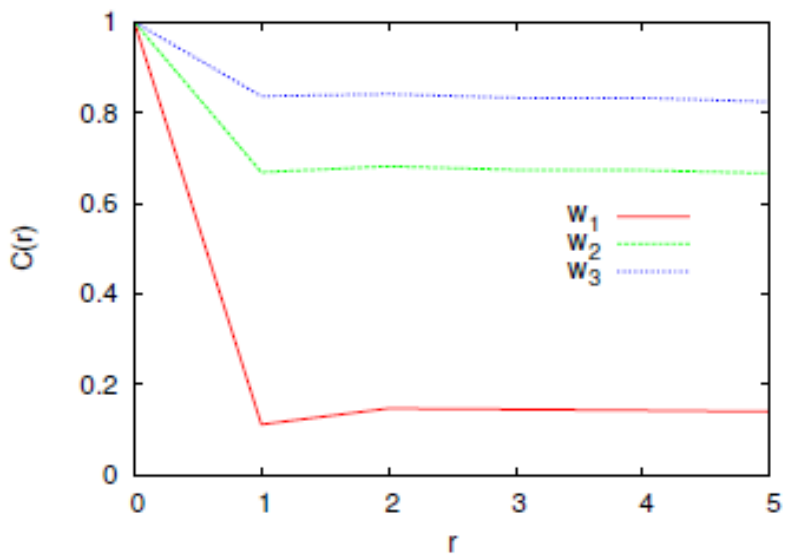

Figure 3: Density-density correlation function versus distance (measured by the neighbourhood, $r=1$ means nearest neighbours, $r=2$ - second NN etc.) from the central plant for three values of water supply. Plant tolerance $\tau=$ 1.0. $w_{1}=0.6, w_{2}=1.0, w_{3}=1.4$. Colour on line.

The presented above plots allow the following description of the basic mechanisms of our model. There is a tendency of plants to adjust their spatial organisation according to the external conditions in order to cope with either surplus or shortage of the resource. This in turn determines whether the elimination will take place at the seedlings or adult plants stage. If $\delta<1$, hence there is a shortage of the resource, eliminated are mostly adult plants. It could be explained as follows. When the resource is scarce, seedlings, with no blocking of water by their NN, are better off than adult plants which lose part of the resource supply. This loss may be crucial and an adult plant could be eliminated before producing seeds. When $\delta>1$, there is too much of the resource and the elimination is at the level of seeds. Once a seed has germinated and developed into an adult plant, its conditions are better since part of the surplus of the resource is blocked. The total success of plants is therefore determined mainly by the success of the adults plants when $\delta<1$ and by the success of seeds when $\delta>1$.

\subsection{Two types of plant}

After showing the basic mechanisms of our model, we may turn to applying them to a more complex case, namely two, quite similar types of annuals, called P1 and P2, differing only by their tolerances, living in the same habitat. We assume that both types are present there from the beginning of the study and no immigrants are allowed.

The only new aspect when considering the system with two types of plant is concerns seeds germination. After the plants have dispersed their seeds, a site may contain $\mathrm{s}_{1}$ seeds coming from P1 and $s_{2}$ seeds coming from P2. Only one seed will be chosen and submitted to the germination test. This seed is selected according to the majority rule, i.e. the chance of choosing, say, a P1 seed, is proportional to the fraction $s_{1} /\left(s_{1}+s_{2}\right)$ of this type of seed on the site. It corresponds to the lottery model with equal weights introduced in (22).

We assume that the initial fractions of the P1 and P2 are equal. Such an assumption allows us to estimate whether a given species contributes more at the end than at the beginning and then we can tell which of the species outperformed the other (17). It corresponds to the average relative growth rate concept (23). We shall call the P1 plants the target plants with fixed value of the tolerance at $\tau_{1}$ $=1.0$ and we shall investigate how the presence of P2 of various tolerances, influences the existence of P1. Fixing the value of $\tau$ and changing only $\tau_{2}$ is reasonable, since we know from the previous section how the dynamics of $\mathrm{P} 1$, living alone, changes with $\tau_{1}$ and moreover the only difference between P1 and P2 are their tolerances. When two types of plant are present, a stationary state is also attained, although it corresponds to just one type of plants alive. The less adapted species are sooner or later eliminated. This may happen after a long time, like 800 years, or just after 50 years and the outcome depends on the supply of the resource and initial arrangement. Elimination of a worse adapted species is a well established fact $(1,24)$. It is of course quite unrealistic to assume that external conditions will remain the same for 800 years. Therefore we have decided to study the case of two types of plant during a period of 100 years. Clearly during that time the system is not in a stationary state but in a transient one. Such a period is still quite long from an experimental point of view. How the picture changes when another time limit is chosen, will be discussed later. The figures for two types of plant for the three successes and the dependence of the densitydensity correlation functions are quite similar to the Figures 2 and 3, and therefore are not presented.

From figure 4 we see that the presence of P2 has a profound effect on P1, restricting their existence range and changing the abundance. There are regions where the better adapted plant eliminates the other one, but there is also an interval of the values of water supply where coexistence is possible. When the tolerances of the species differ considerably, like for $\tau_{1}=1.0$ and $\tau_{2}=$ 0.6 , in the upper left panel, the coexistence zone is rather small. Better suited species have a large advantage over the others and eliminate them. When however the species features are simiar, they could coexist over a relatively large region of water supply. How the target plants (P1 with $\tau_{1}=$ 1.0) are affected by the presence of $P 2$ plants with different tolerances, is shown in figure 5 where we compare the number of plants P1 when they grow without P2 (the mono curve) and when P2 are 

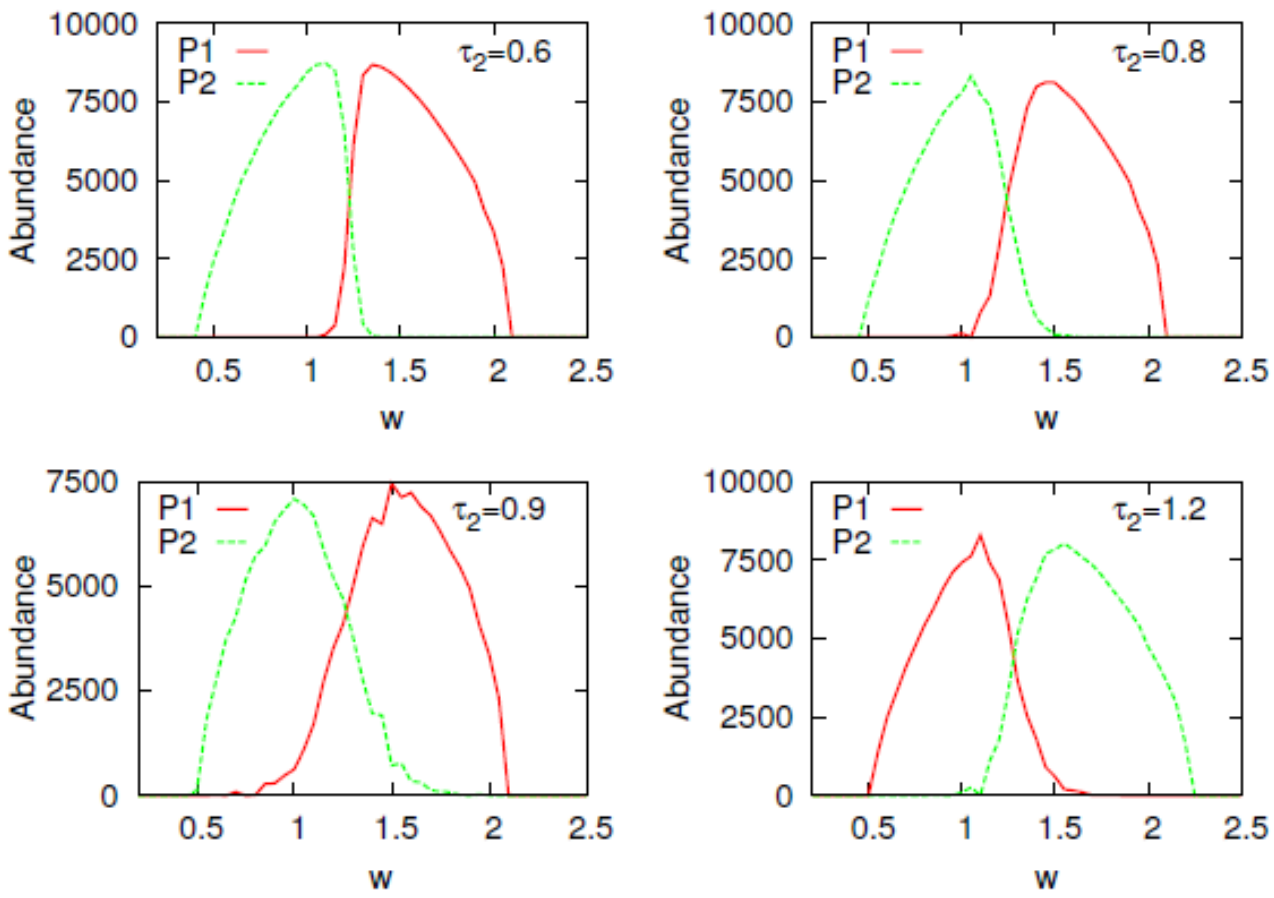

Figure 4: Number of P1 and P2 plants for various tolerances of P2 and as a function of the water supply. $\tau_{1}$ is everywhere equal 1.0. $\tau_{2}$ is as shown on the figures. Colour on line.

present and have various tolerances. Several features should be noticed. When $\tau_{2}=1.2$ the P1 are completely unaffected for small values of water, since there the advantage of P1 is so large that P2 are eliminated quite quickly. Once however the P2 are able to survive, they reduce the number of P1 and with decreasing $\mathrm{w}$ the process of replacing P1 by P2 is more and more quickly. If $\tau_{2}=0.8$, the P2 appear at smaller values of $\mathrm{w}$ than when $\tau_{2}=1.2$, but could eliminate P1 only when $\mathrm{w}$ is quite small. As with the $R^{*}$ level of Tilman (25), the species with lower value of $\tau$ will eliminate the others when $\delta<1$, while for $\delta>1$ the other species will win.

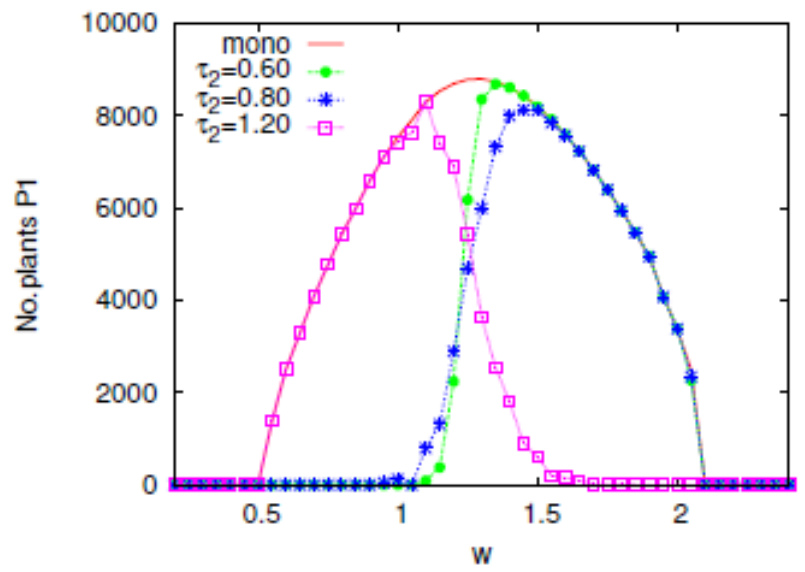

Figure 5: Number of P1 where they grow alone (mono curve) and with P2 of various tolerances as a function of the water supply. $\tau_{1}=1.0$ for all cases. Colour on line.

This figure gives an answer to the question often asked by ecologists (17) - how much the target species' performance (here the number of plants) is attributable to the presence of other species. The figure shows how this influence changes with the supply of the resource and the relation between tolerances of the two species.

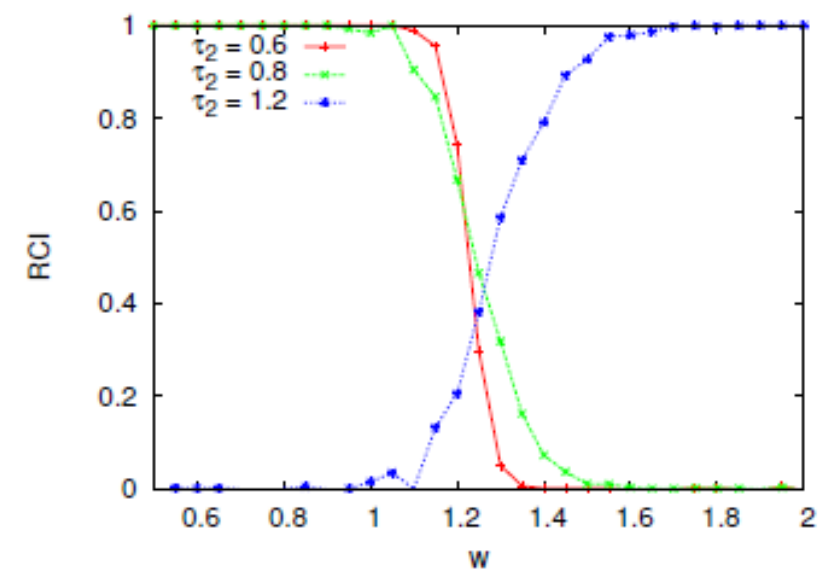

Figure 6: The RCI index, for definition see the text, as a function of the water supply and several values of the P2 plants tolerance. Colour on line.

When two species coexist and compete for a limited resource, often a plant competition index is introduced. There is however still a controversy at to how it should be defined. In a paper by Weigelt and Jolliffe (26) nearly 50 of them have been described and discussed. In view of the controversy we have chosen a simple one, namely the Relative Competition Index (RCI), introduced in (27) as

$$
R C I=\frac{N P_{\text {mono }}-N P_{\text {mix }}}{N P_{\text {mono }}}
$$



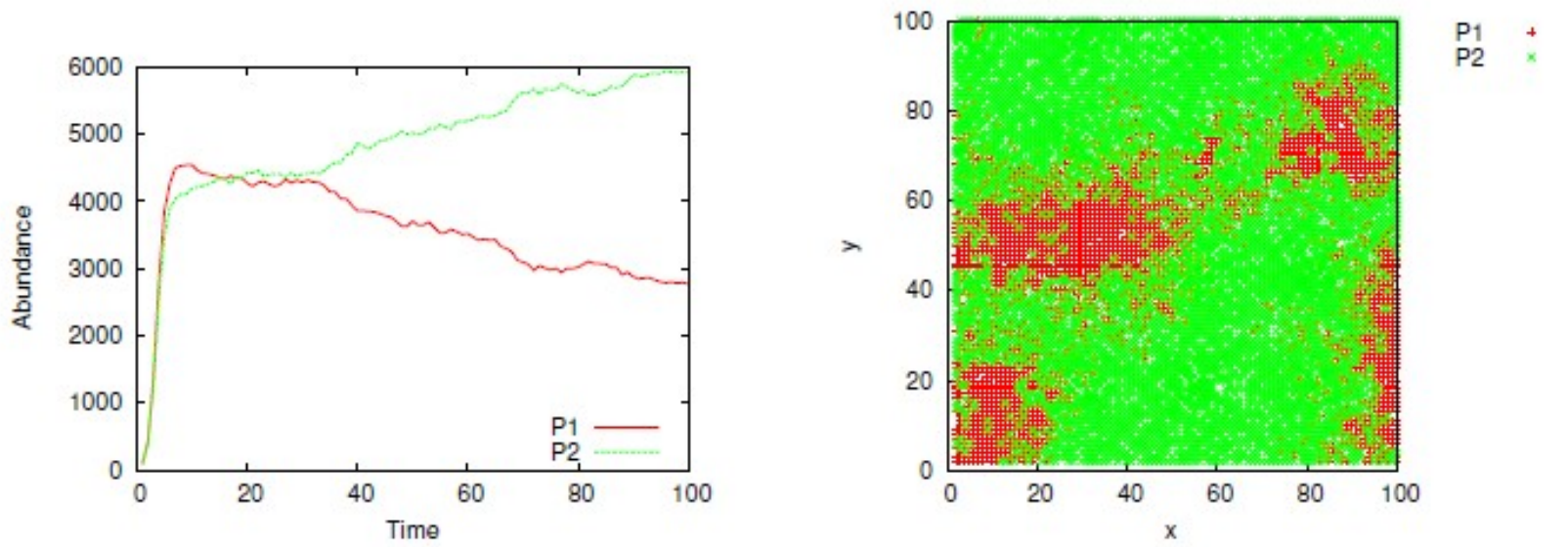

Figure 7: Temporal dependence of the abundances of plants P1 and P2 (left panel). Spatial arrangement of the P1 (red crosses) and P2 (green circles) plants at the end of simulation, i.e. after 100 years (right panel). $w=1.3, \tau_{1}=1.0$ and $\tau_{2}=0.8$. Colour on line.
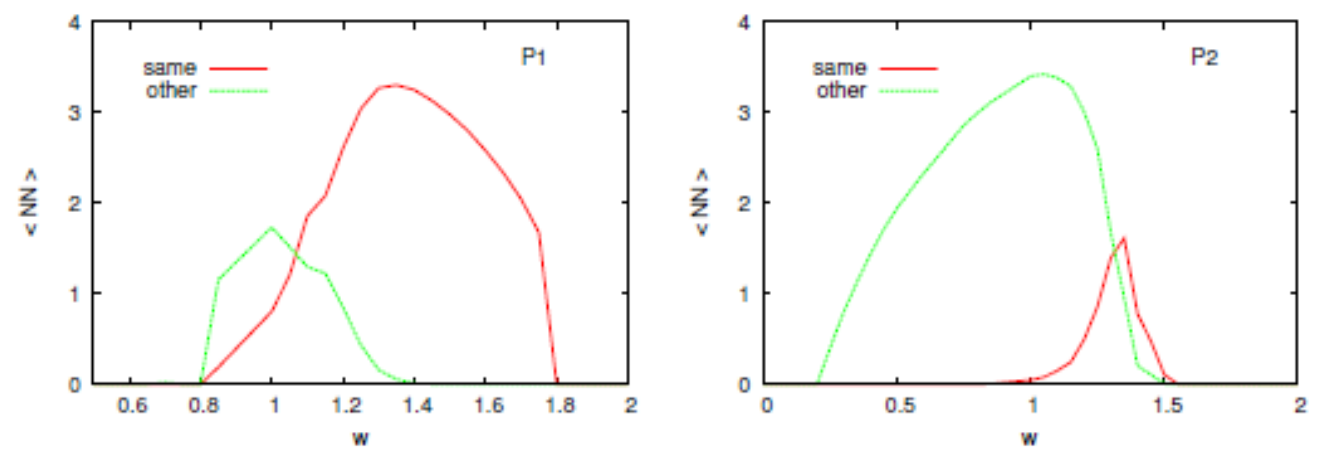

Figure 8: Average number of nearest neighbours, as functions of the water supply w, of the same type (same) and of the other (other) types. $\tau_{1}=1.0, \tau_{2}=0.9$. Left panel P1 plants, right panel P2 plants. Colour on line.

where $\mathrm{NP}_{\text {mono }}$ is the number of plants in a monoculture and $\mathrm{NP}_{\text {mix }}$ is the number of the same type of plants living with other plants. In figure 6 the RCI is presented as a function of the water supply $\mathrm{w}$ and several values of $\tau_{2}$. There is no competition when RCI is either zero or when RCI = 1 , when the worse adapted plants cease to exist. The region in between marks the competition and coexistence range. If $\mathrm{RCI} \leq 0.5$ the $\mathrm{P} 1$ are dominant, otherwise $\mathrm{P} 2$ are more abundant.

An interesting problem, which could be studied only via computer simulations in agent based models, is the spatial organisation of the two populations. Figure 7 shows in the left panel the temporal dependence of the abundance of P1 and P2 plants for the following values of the parameters: $w=1.3, \tau_{1}=1.0, \tau_{2}=0.8$. In the right panel we present the spatial distribution of plants at the end of simulation. The figures represent one simulation (without averaging), but general features are quite similar for other realisations. We see from that figure that even in a homogeneous habitat with only one type of resource and plants differing by the tolerance to surplus of the resource, plants of the same type have a tendency to grow in clusters. Even without any kind of a trade-off plants are able to divide the habitat into their own niches. It should be noted that the time evolution of two competing plants, shown in the left panel of figure 7, resembles plots describing similar process in (3).

There are several ways to measure this clustering effect. One of them is presented in Figure 8 as the average number of nearest neighbours (NN) of the same type as the central plant (same line) and of the other type (other line). Left panel shows this dependence for the P1 plants and the right hand panel - for P2 plants.

Finally, we discuss how the results presented above change when the parameters we have fixed assume another values. Since the plots for the two types of plants were obtained for a transient regime, it is important to check how the picture will change were we to take another time limit at which the plants' abundances were calculated. 100 years taken by us in the simulation, is a long period and therefore we have repeated the simulations for 50 years time limit. There are some, but quite minor, differences between the results for the two time limits. We may therefore conclude that in the transitory regime at which we investigate the population evolution, the results do not change qualitatively when the time limit varies within, say, the interval [50 - 150] years and in this respect the results are generic. Changes to the population size in time can be followed in figure 7 on left panel. 
The size of the system $L=100$ is a compromise between execution speed and avoiding stochastic extinctions and poor statistics. For small systems, such as $L=20$, even local demographic stochasticity could, in the case of non-linear dynamics, lead to global effects, like extinction of species $(1,28)$.

Another parameter which could influence the dynamics of plants in our model is $\alpha$ - the "interaction strength" describing the effect on a plant of its NN. When $\alpha=0$ we have a system of completely independent plants when the neighbourhood has no influence on the central plant. The main difference among plots for various values of $\alpha$ is the shift of the maximum number of plants towards larger values of $w$ when $\alpha$ is growing, since with increasing $\alpha$ more and more of the resource is blocked by the NN. The second effect of growing $\alpha$ is pushing down the maximum abundance. This comes from the fact that for large $\alpha$ the nearest neighbourhood plays a more important role and since not all the plants have the same neighbourhood, optimal conditions are more widely spread than for small values of $\alpha$. Also the coexistence region shrinks with growing $\alpha$. For $\alpha=0$ the dependence of the number of plants on water supply has a very sharp maximum at $w=\delta$.

When the maximum number of seeds a plant can produce in optimal condition (parameter $\beta$ ) is small, such as 3 , populations could be eliminated quite quickly. Increasing $\beta$ to larger than 6 values, has practically no effect, since from each site only one seed is taken for the germination test.

\section{Mean Field}

A mean field approach is very often used in ecology $(1,3,29)$ and it is therefore instructive to show on our simple model when simplifications made in the mean field approach could be accepted. In our model instead of dealing with individual plants we may use global observables, such as the density $\rho(t)$ of plants at time $t$ and the rates will replace chances. The survival rate tells us what fraction of the plants' population survived till the moment they could produce seeds. The germination rate determines how many of the seeds will germinate. Keeping the same notation and assuming, as before, that the plants interact only with their nearest neighbours with strength $\alpha$ and that each plant can produce at most $\gamma$ seeds, we get the following coupled equations determining the dynamics of the density of plants $\mathrm{P} 1$ and $\mathrm{P} 2$

$$
\begin{aligned}
& \rho_{1}(t)=\rho_{1}(t-1)\left[(1-\rho(t-1)) \cdot \gamma \cdot x_{11} \cdot x_{12}\right] \\
& \rho_{2}(t)=\rho_{2}(t-1)\left[(1-\rho(t-1)) \cdot \gamma \cdot x_{21} \cdot x_{22}\right]
\end{aligned}
$$

where $\rho(t)$ is the sum of the densities of both types of plant and $\rho_{\mathrm{m}}$ is the density of plants of type $m$. The first term in the square brackets on the right hand side corresponds to the Verhulst factor reducing, otherwise unlimited, growth of the population. $X_{\mathrm{m} 1}$ and $X_{\mathrm{m} 2}$ are the survival and germination rates, respectively, of the plants $m$. The maximum number of seeds $\gamma$ used here is not equal to the parameter $\beta$ used in the simulations, as there we took only one seed from each site. Analogously as in the simulations, we may define the quantity $\Delta$, which determines the amount of water available to a plant

$$
\Delta(t)=w(1-4 \cdot \alpha \cdot \rho(t))
$$

The factor 4 comes from the number of NN. As before, depending on whether $\Delta$ is smaller or larger than 1, we use two forms of the survival rate $x_{11}$ for the P1 plants with tolerance $\tau_{1}$

$$
X_{11}=\left\{\begin{array}{lc}
\exp \left(-(1-\Delta)^{2} \cdot \tau_{1} / \Delta\right) & \text { if } \Delta<1 \\
\exp \left(-(1-\Delta)^{2} /\left(\tau_{1} \cdot \Delta\right)\right) & \text { if } \Delta<1
\end{array}\right.
$$

The germination, $\mathrm{x}_{12}$, rate has the same form as the one above, with $w$ replacing $\Delta$, since there is no blocking affect for the seedlings. Formulae for the P2 plants are the same with $\tau_{2}$ in place of $\tau_{1}$.

Using these equations we get the densities of one type of plants as a function of the water supply $w$ for 3 values of the tolerance, shown in figure 9 for $\alpha=0.1$ and $\gamma=2.5$.

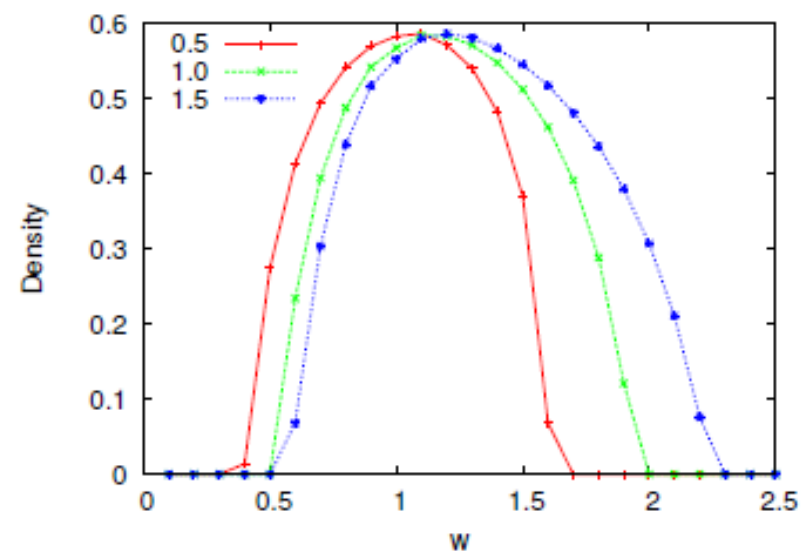

Figure 9: Densities of one type of plants versus water supply $\mathrm{w}$ for three values of the tolerance obtained from mean field type calculations. $\tau_{1}=1$. Colour on line.

As we can see, the plots are very similar to what we obtained from simulation, viz. figure 1 . For two types of plant we get the figure 10, corresponding to figure 4. Again, the behaviour is very similar, including the coexistence zones. It should however be stressed that while construction of a mean field type model is much simpler and obtaining the results is much quicker, 

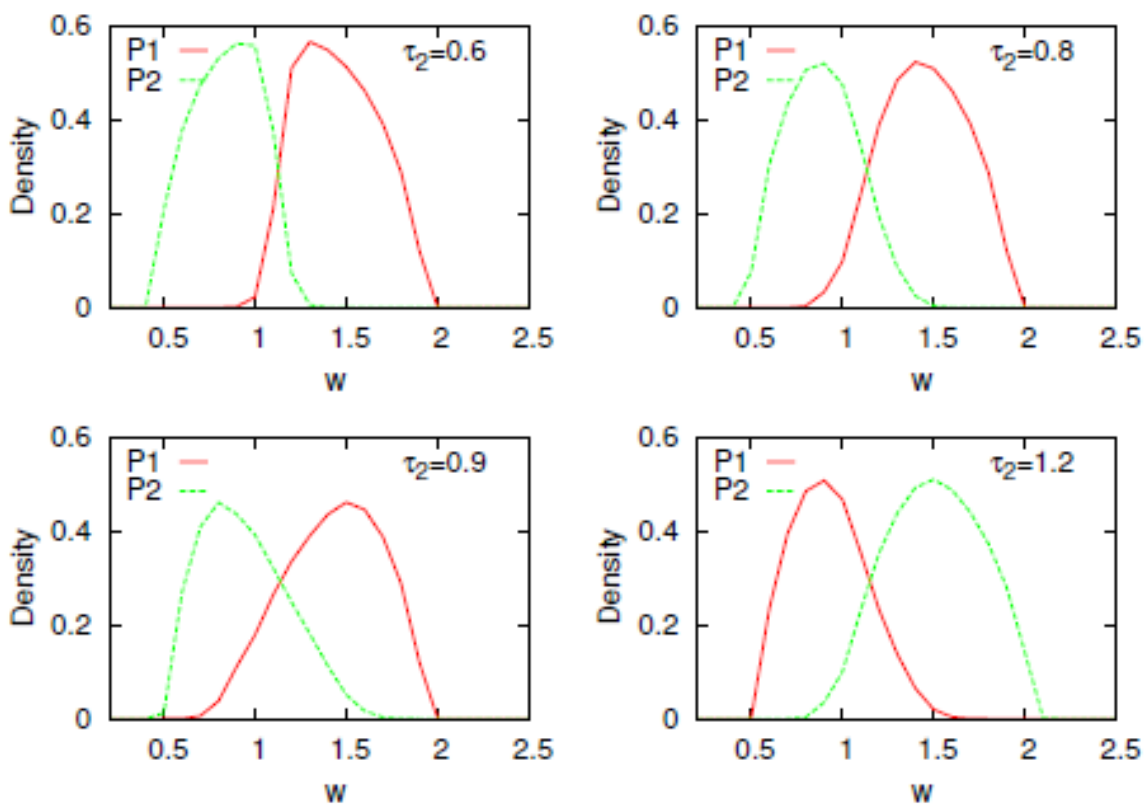

Figure 10: Densities of two types of plant versus water supply w for four values of the tolerance of plants P2 obtained from mean field type calculations. Colour on line.

yet it is impossible to see what kind of a spatial structure (if any) the described population forms, or what are the correlations between the same or different types of plant. To this end, spatial explicit, or agent-based models are necessary.

\section{Conclusions and Discussion}

We have presented and discussed a computerbased simulation model describing life cycles of a population of annual plants competing for a single resource. In most papers dealing with coexistence of species some kind of a trade-off $(3-5,29)$ or heterogeneity in space or time $(1,8,22)$ is assumed. In the coexistence models (1) species have different inter- and intra-specific competition coefficients, while in the niche theory (25) species coexistence is linked with different competition capabilities for different resources. In our model we have no such elements as the habitat is homogeneous, there is only one resource and plants differ just by their tolerance to the resource supply. One type better supports shortage of water, while the other tolerates better too much of it. Taking into account that plants suffer from both - shortage of water as well of too much of it, is another novel feature of our model.

The questions we have asked are - is a coexistence of two different species possible in such a simple system, without the competitioncolonisation trade-off $(5,6,12)$ or disturbances $(1$, $10,11)$ ? If so, how the dominance of one species depends on the supply of the resource and the difference in the tolerances. How does the presence of a second type of plants, with quite similar characteristics, affect the target plants? What will be the spatial structure in the coexisting phase? To the best of our knowledge such investigations have not yet been carried out.
The results obtained from the simulations and presented in the Results section, allow us to conclude that our simple model leads to a coherent description of the situation and brings new light on the basic questions listed above.

While the system of one type of plants arrives at a stationary state very quickly (after just 10 years), dynamics of two types of plant is by far more complex. The final state, which could be attained sometimes only after several hundred years, is always a singlespecies state. In the transient period coexistence, lasting also sometimes very long, is possible, depending on the random initial conditions. The range of values of the resource over which it happens, is larger if the tolerances do not differ much and happens where the supply is close to the demand. The life cycle of a plant could be controlled either by elimination of the seedlings (including germination blocking) or by elimination of adults plants. We have shown that it depends on the supply of the resource which of the mechanism is more important. The value of the tolerance does not play a significant role here.

Using an individual-based model allowed us to determine also spatial organisation of plants. We have shown that depending on the availability of the resource the plants have a tendency to stay away one from another when the resource is scarce. When there is an adequate amount of it, there is no visible pattern and when there is too much of the resource, the plants have a tendency to form dense clusters. In a system of two types of plant they form groups of alike plants and this tendency grows when the difference in the two tolerances becomes smaller.

We have shown that even in a homogeneous system with plants competing for the same 
resource and without any immigrants, coexistence of two types of plant is possible. Each species creates its own niche where only a given type of plant is growing, pushing out the other type. It shows that the niche theory could be applied also in a homogeneous system with one resource.

We have demonstrated that a much simpler mean field type approach to the coexistence problem could yield correct results regarding the conditions for such coexistence, however it can tell us nothing about the spatial structure of the two populations, in particular, whether the two types of plant created their own niches.

Once the basic mechanisms of such a system are understood, it would be possible to extend the model by considering different size of the plants (30), competition for more than one resource (31), different maximum number of seeds and their size (12), effect of litter (32) other resources $(33,34)$. Also spatial and/or temporal inhomogeneities either in the form of cyclic or random changes (9) could be incorporated.

\section{Acknowledgements}

We are very grateful for Monica Slowikowska for reading and correcting the manuscript.

\section{Conflict of Interest}

The authors declare no conflict of interest.

\section{Authors' Contribution}

MD did the calculations for the mean field. AP did the simulations. The text was written in close collaboration.

\section{References}

[1] Chesson P. Mechanisms of maintenance of species diversity. Annual Review of Ecology and Systematics 2000;31:343-366.

[2] Levins R, Culver D. Regional coexistence of species and competition between rare species. Proceedings of the National Academy of Sciences 1971;6:1246- 1248 .

[3] Tilman D. Competition and biodiversity in spatially structured habitats. Ecology 1994;75:2-16. DOI: $10.2307 / 1939377$

[4] Holmes E, Wilson H. Running from trouble: long distance dispersal and the competitive coexistence of inferior species. American Naturalist 1998;151:578- 586. DOI:10.1086/286143

[5] $\mathrm{Yu} \mathrm{D}$, Wilson $\mathrm{H}$. The competition-colonization trade-off is dead; long live the competitioncolonization trade-off. The American Naturalist 2001;158:49-63.

[6] Coomes D, Rees M, Turnbull L, Ratcliffe S. On the mechanisms of coexistence among annual-plant species, using neighborhood techniques and simulation models. Plant Ecology 2002;163:23-39. DOI: $10.1086 / 286143$

[7] Tilman D. The importance of the mechanisms of interspecific competition. American Naturalist 1987;128:769-774. DOI:10.1086/286143
[8] Pacala S. Neighborhood models of plant population dynamics. 2. multi-species models of annuals. Theoretical Population Biology 1986;29(2):262-292.

[9] Roxburgh S, Shea K, Wilson J. The intermediate disturbance hypothesis: patch dynamics and mechanisms of species coexistence. Ecology 2004;85(2):359-371. DOI: 1890/03-0266

[10] Miller A, Reilly D, Bauman S, Shea K. Interactions between frequency and size of disturbance affect competitive outcomes. Ecological Research 2012;27(4):783-791. DOI: 10.1007/s11284-012-054-4

[11] Seifan M, Seifan T, Jeltsch F, Tielbörger K. Combined disturbances and the role of their spatial and temporal properties in shaping community structure. Perspectives in Plant Ecology, Evolution and Systematics 2012;14(3):217229.

[12] Muller-Landau H C. The tolerance-fecundity tradeoff and the maintenance of diversity in seed size. Proceedings of the National Academy of Sciences 2010;107(9):4242-4247.

[13] Goldberg D, Fleetwood L. Competitive effect and response in four annual plants. Journal of Ecology 1987;73(4):1131-1143. DOI: $10.2307 / 2260318$

[14] Grime J. Evidence for the existence of three primary strategies in plants and its relevance to ecological and evolutionary theory. American Naturalist 1977;111:1169-1194.

[15] Keddy P, Shipley B. Competitive hierarchies in herbaceous plant communities. Oikos 1989;54:234241. DOI: $10.1086 / 286143$

[16] Tilman D. Mechanisms of plant competition for nutrients: the elements of a predictive theory of competition. Perspectives on Plant Competition 1990;117-141.

[17] Connolly J, Wayne P, Bazzaz F. Interspecific competition in plants: how well do current methods answer fundamental questions? American Naturalist 2001;157(2):107-125. DOI: $10.1086 / 318631$

[18] Hickman J. Energy allocation and niche differentiation in four co-existing annual species of polygonum in western north America. Journal of Ecology 1977;65:317-326. DOI: 10.2307/2259080

[19] Reynolds D. Populational dynamics of three annual species of alpine plants in the rocky mountains. Oecologia 1984;62(2):250-255.

[20] Schenk H. Root competition: beyond resource depletion. Journal of Ecology 2006;94(4):725-739. DOI: $10.111 /$ j.1365-2745.2006.01124.x

[21] Shirley $\mathrm{H}$. The influence of light intensity and light quality upon the growth of plants. American Journal of Botany 1929;16:354-390.

[22] Chesson P, Warner R. Environmental variability promotes coexistence in lottery competitive systems. American Naturalist 1981;117(6):923-943.

[23] Evans G. The quantitative analysis of plant growth Vol 1. Univ of California Press; 1972

[24] Levin S. Community equilibria and stability, and an extension of the competitive exclusion principle. American Naturalist 1970;104:413-423. DOI: $10.1086 / 28.2676$

[25] Tilman D. Resource competition and community structure. Princeton University Press, Princeton, New Jersey; 1982 
[26] Weigelt A, Jolliffe P. Indices of plant competition. Journal of Ecology 2003;91(5):707-720. DOI: 10.1046/j.1365-2745.2003.00805.x

[27] Grace J. On the measurement of plant competition intensity. Ecology 1995;76:305-308. DOI: $10.2307 / 1940651$

[28] Durrett R, Levin S. The importance of being discrete (and spatial). Theoretical Population Biology 1994;46(3):363-394.

[29] Letten A D, Ku P J, Fukami T. Linking modern coexistence theory and contemporary niche theory. Ecological Monographs 2017;87(2):161-177

[30] Stein A, Gerstner K, Kreft H. Environmental heterogeneity as a universal driver of species richness across taxa, biomes and spatial scales. Ecology Letters 2014;17(7):866-880. DOI: 10.1111/ele.12277
[31] Diàz-Sierra R, Zavala $M$, Rietkerk M. Positive interactions, discontinuous transitions and species coexistence in plant communities. Theoretical Population Biology 2010;77(2):131-144. DOI: 10.1016/j.tpb.2009.12.001

[32] Kącki Z, Pękalski A. The impact of competition and litter accumulation on germination success in a model of annual plants. Physica A 2011;390:25202530. DOI: 10.1016/j.physa.2011.03.014

[33] Pękalski A, Szwabiński J. Dynamics of three types of annual plants competing for water and light. Physica A 2013;392:710-721. DOI: 10.1016/physa.2012.09.029

[34] Droz M, Pękalski A. Model of annual plants dynamics with facilitation and competition. Journal of Theoretical Biology 2013;335:1-12. DOI: 10.1016/j.jtbi.2013.06.010 